\title{
ON TOPOLOGICAL TYPES OF REDUCED SEXTICS
}

\author{
Masaharu Ishikawa, Tu Chanh Nguyen, and Mutsuo Oka
}

\begin{abstract}
We present a computational method for obtaining generic forms of sextics with a given configuration of local singularities. Using this method, we first complete the classification of topological types of local singularities appearing on reduced sextics. Next we give the list of possible configurations of singularities containing at least one non-simple singularity and satisfying $\rho(5) \geq 7$, and show that such a sextic is of torus type, which has been conjectured by the third author.
\end{abstract}

\section{Introduction}

Our main interest in this paper is to study the topological types of singularities appearing on reduced sextics and their configurations.

In the first half of this paper ( $\S 3)$, we study the topological types. By the works of Urabe, Yoshihara, Yang [U, Yo, Ya], it is known that all simple singularities with Milnor number less than or equal to 19 can appear on sextics. On the other hand, Degtyarev proved in [D] that the rigid isotopy type of an irreducible sextic with at least one non-simple singularity is determined by the configuration of singularities of the sextic and Wall listed in [W] all the possible non-simple singularities on reduced sextics using the notation of Arnold [AGV].

It is well-known that the topological type of an isolated singularity is determined by its Newton boundary if the boundary is Newton non-degenerate. We focus on this property and give the classification of singularities appearing on reduced sextics in terms of Newton boundaries. It turns out that the singularities are Newton non-degenerate except the series of classes of singularities $S_{n}, n=1,2, \ldots, 6$. Note that our classification is independent of the work of Wall, though our list obtained is, of course, identical to his list.

Our strategy is computational. We start from the generic form of a sextic

1991 Mathematics Subject Classification. 14H30, 14H45, 32S55.

Key words and phrases. non-degenerate, germs of singularities.

The first author is partially supported by JSPS (Japan Society for the Promotion of Science) Research Fellowships for Young Scientists and the second author is supported by JSPS Postdoctoral Fellowships.

Received October 14, 2003; revised July 23, 2004. 


$$
f(x, y)=\sum_{i+j \leq 6} a_{i j} x^{i} y^{j} .
$$

Assume that the origin $(0,0)$ is a singularity and put $a_{00}=a_{10}=a_{01}=0$. We take a series of changes of local coordinates $\left(x_{j}, y_{j}\right)$ as long as the Newton number $v\left(\Gamma_{-}\left(f ;\left(x_{j}, y_{j}\right)\right)\right)$ is strictly greater than the previous one $v\left(\Gamma_{-}\left(f ;\left(x_{j-1}, y_{j-1}\right)\right)\right)$. As the Newton number is bounded above by the Milnor number, this process stops after a finite number of operations. When we arrive at the coordinate system $\left(x_{\tau}, y_{\tau}\right)$ for which no further change of coordinates is possible, we check if the polynomial is reduced, if the Newton boundary is nondegenerate and if it really exists. The classification of local singularities of reduced sextics is completed by checking all possible trees of the changes of coordinates. This classification is done in Section 3.

In the second half $(\S \S 4,5)$, we deal with reduced sextics having special configurations and observe if they are of torus type (see Section 4 for the definition of torus type).

In Section 4 we prove that a reduced sextic with configuration of singularities on a tame sextic of torus type and having at least one non-simple singularity is always of torus type (Theorem 12).

In Section 5 we compute the $\rho$-invariants of non-simple singularities appearing on reduced sextics and prove, using the result in Section 4 and the discussion in $[\mathrm{W}]$, that if a sextic has at least one non-simple singularity and satisfies $\rho(5) \geq 7$ then it is of torus type (Theorem 16), which has been conjectured by the third author.

\section{Preliminaries}

2.1. Notations. We use the following standard notations for simple singularities:

$$
\left\{\begin{array}{l}
A_{n}: x^{2}+y^{n+1}=0 \quad(n \geq 1), \\
D_{n}: x^{2} y+y^{n-1}=0 \quad(n \geq 4), \\
E_{6}: x^{3}+y^{4}=0, E_{7}: x^{3}+x y^{3}=0, E_{8}: x^{3}+y^{5}=0,
\end{array}\right.
$$

where the equations written in the right-hand side are the normal forms of these singularities (cf. Remark 2 below). Furthermore, we use the following notations for non-simple singularities as their normal forms:

$$
\left(\begin{array}{l}
B_{p, q}: x^{p}+y^{q}=0 \quad(p \leq q), \\
s B_{p, q}: s\left(x^{p}+y^{q}\right)=0, s=x, y, x y \quad(p \leq q), \\
C_{p, q}: x^{p}+y^{q}+x^{2} y^{2}=0 \quad(2 / p+2 / q \leq 1, p \leq q), \\
y C_{p, q}: y\left(x^{p}+y^{q}+x^{2} y^{2}\right)=0 \quad(2 / p+2 / q \leq 1, p \leq q), \\
D_{p, q}: x^{p}+y^{q}+x^{2} y^{3}=0 \quad(2 / p+3 / q \leq 1), \\
F_{p, q}: x^{p}+y^{q}+x^{2} y^{3}+x^{3} y^{2}=0 \quad(6 \leq p \leq q), \\
S_{n}:=T_{n-1} \backslash T_{n} \quad(n=1, \ldots, 6), \text { where }
\end{array}\right.
$$




$$
\mid \begin{aligned}
& T_{0}:=\left(x^{2}-y^{3}\right)^{2}+\sum_{0<i+j \leq 6,3 i+2 j>12} a_{i j} x^{i} y^{j}=0, \\
& T_{i}:=\left\{R_{1}=\cdots=R_{i}=0\right\}, \\
& R_{1}:=a_{15}+a_{32}, R_{2}:=4 a_{24}+4 a_{41}-a_{32}^{2}, R_{3}:=2 a_{33}+2 a_{50}-a_{32} a_{41}, \\
& R_{4}:=-4 a_{42}+2 a_{32} a_{50}+a_{41}^{2}, R_{5}:=2 a_{51}-a_{41} a_{50} \text { and } R_{6}:=-4 a_{60}+a_{50}^{2} .
\end{aligned}
$$

These notations of the singularities are to be understood in the sense of topological equivalence. The notations $S_{3}$ and $S_{6}$ are denoted as $S p_{1}$ and $S p_{2}$ in $[\mathrm{Ph}]$ respectively. All other singularities appearing in $[\mathrm{Ph}]$ have the same notations. Note that the Milnor numbers $\mu$ of these singularities are given by the following formulae:

$$
\begin{gathered}
\mu\left(B_{p, q}\right)=(p-1)(q-1), \quad \mu\left(x B_{p, q}\right)=p q-p+q, \quad \mu\left(y B_{p, q}\right)=p q+p-q, \\
\mu\left(x y B_{p, q}\right)=(p+1)(q+1), \quad \mu\left(C_{p, q}\right)=p+q+1, \quad \mu\left(y C_{p, q}\right)=3 p+q, \\
\mu\left(D_{p, q}\right)=2 p+q+1, \quad \mu\left(F_{p, q}\right)=p+q+6, \quad \mu\left(S_{n}\right)=15+n .
\end{gathered}
$$

Remark 1. The correspondence between our notations and those in [W] (cf. $[\mathrm{AGV}]$ ) is described in the following table (where $i \geq 0$ and $j, k \geq 1$ ):

\begin{tabular}{|c|c|c|c|c|c|c|c|c|c|}
$D_{4+i}$ & $B_{3,3 k}$ & $B_{3,3 k+1}$ & $B_{3,3 k+2}$ & $B_{4,4}$ & $B_{4,5}$ & $B_{4,6}$ & $B_{5,5}$ & $B_{5,6}$ & $B_{6,6}$ \\
\hline$E_{1, i}$ & $E_{k, 0}$ & $E_{k,(-1)}$ & $E_{k,(1)}$ & $X_{1,0}$ & $W_{12}$ & $W_{1,0}$ & $N A_{0,0}$ & $N F_{20}$ & none
\end{tabular}

\begin{tabular}{|c|c|c|c|c|c|c|c|c|c|c|}
$y B_{3,4}$ & $y B_{3,5}$ & $y B_{3,6}$ & $x B_{3,4}$ & $y B_{4,5}$ & $x B_{2,5}$ & $x B_{3,5}$ & $x B_{4,5}$ & $x B_{2,7}$ & $x y B_{2,3}$ & $x y B_{3,4}$ \\
\hline$Z_{11}$ & $Z_{13}$ & $Z_{1,0}$ & $W_{13}$ & $N C_{19}$ & $E_{13}$ & $W_{17}$ & $N F_{21}$ & $E_{2,(0)}$ & $Z_{12}$ & $N C_{20}$
\end{tabular}

\begin{tabular}{|c|c|c|c|c|c|c|c|c|c|}
$C_{3,6+k}$ & $C_{4+j, 4+k}$ & $y C_{3,6+k}$ & $y C_{5,4+k}$ & $D_{3,9+k}$ & $D_{4,7}$ & $D_{5,5+k}$ & $D_{6,5+i}$ & $F_{5+j, 5+k}$ & $S_{k}$ \\
\hline$E_{2, k}$ & $T_{2,4+j, 4+k}$ & $Z_{1, k}$ & $N B_{(0)}^{k}$ & $E_{3, k}$ & $W_{1,1}$ & $N A_{k, 0}$ & $N B_{(-1)}^{i}$ & $N A_{j, k}$ & $W_{1, k}^{\#}$
\end{tabular}

2.2. Newton boundary and coordinate changes. For a given analytic function $f(u, v)=\sum_{i, j} a_{i j} u^{i} v^{j}$, we denote by $\Gamma(f ;(u, v))$ the Newton boundary with respect to the coordinates $(u, v)$. We may abbreviate it to $\Gamma(f)$ if the choice of the coordinates is clear. The cone of $\Gamma(f ;(u, v))$ with the origin is denoted by $\Gamma_{-}(f ;(u, v))$. The Newton principal part of $f$ is defined by $\operatorname{NPP}(f):=$ $\sum_{(i, j) \in \Gamma(f)} a_{i j} u^{i} v^{j}$. The Newton number $v(\Gamma(f))$ is defined by the alternating sum

$$
v(\Gamma(f)):=2 \text { volume } \Gamma_{-}(f)-(a+b)+1,
$$

where $a$ (resp. $b)$ is the length of the face of $\Gamma_{-}(f)$ on the $u$-axis (resp. $v$-axis). For example, $a$ is given by $\min _{i \in \mathbf{N}}\left\{i \mid a_{i 0} \neq 0\right\}$. If no such monomial exists, $a=0$ by definition.

Let $\Delta$ be a face of the Newton boundary. The polynomial $f_{\Delta}(u, v):=$ $\sum_{(i, j) \in \Delta} a_{i j} x^{i} y^{j}$ is called the face function. Let $p, q$ be the pair of coprime positive integers such that ${ }^{t}(p, q)$ is a normal vector to $\Delta$. Since $\Delta$ is included in 
the line $\left\{(i, j) \in \mathbf{R}^{2} \mid p i+q j=d\right\}$, where $d$ is some positive integer, $f_{\Delta}(u, v)$ is a weighted homogeneous polynomial of type $(p, q ; d)$ and uniquely factorized as $f_{\Delta}(u, v)=c u^{a} v^{b} \prod_{i=1}^{\ell}\left(v^{p}-\xi_{i} u^{q}\right)^{v_{i}}$, where $\xi_{1}, \ldots, \xi_{\ell}$ are mutually distinct non-zero complex numbers. If $f_{\Delta}$ has no multiple factor (i.e., $v_{i}=1$ for each $i$ ), we say that $f$ is non-degenerate on $\Delta$. Otherwise $f$ is said to be degenerate on $\Delta$. If $f$ is non-degenerate on all faces, we say that $f$ is non-degenerate or more precisely Newton non-degenerate.

For a given weight vector $Q={ }^{t}(p, q)$, we denote the lowest terms with respect to the weight $Q$ by $f_{Q}(x, y)$. Let $d(Q)$ denote the minimal degree with respect to $Q$ and set $\Delta(Q ; f):=\{p i+q j=d(Q)\} \cap \Gamma(f)$. Then $f_{Q}(x, y)$ is the sum of $a_{i j} x^{i} y^{j}$ for $(i, j) \in \Delta(Q ; f)$. In particular, if $Q$ is a normal vector to a face $\Delta$ then $\Delta(Q ; f)=\Delta$ and $f_{Q}(x, y)=f_{\Delta}(x, y)$. In this case we may use both of the notations $f_{Q}(x, y)$ and $f_{\Delta}(x, y)$ for representing the face function.

It is well-known that the topological type of the germ of an isolated singularity $f(u, v)=0$ with non-degenerate Newton boundary depends only on the Newton boundary $\Gamma(f ;(u, v))$. See $[\mathrm{K}, \mathrm{O} 2]$ for further details.

When we are given a polynomial of $f(x, y)$, for the determination of the normal forms we use two types of coordinate changes.

(i) A change of coordinates given by

$$
(x, y) \mapsto(a x+b y, c x+d y),
$$

where $a, b, c, d \in \mathbf{C}$ and $a d-b c \neq 0$. This is a linear coordinate change. It does not change the degree of a given polynomial.

(ii) A change of coordinates given by either

or

$$
(x, y) \mapsto\left(x, y+s_{1} x+s_{2} x^{2}+\cdots+s_{p} x^{p}\right)
$$

$$
(x, y) \mapsto\left(x+t_{1} y+t_{2} y^{2}+\cdots+t_{q} y^{q}, y\right),
$$

where $s_{i}, t_{j} \in \mathbf{C}, s_{p} \neq 0$ and $t_{q} \neq 0$. This is called a triangular coordinate change. Note that a triangular coordinate change may change the degree of a given polynomial.

Remark 2. The normal forms presented in Section 2.1 are Newton nondegenerate except for $S_{1}, \ldots, S_{6}$. Thus if a face has other integral point $(a, b)$ on $\Gamma(f)$, the monomial $c x^{a} y^{b}$, with $c \in \mathbf{C}$ generic, can be added. If $(1, b)$ is on $\Gamma(f)$, the monomial on $y$-axis can be chopped off (a negligible truncation in the sense of [O]). For example, for $n=2 m+1$ odd, $A_{n}$ and $D_{n+1}$ can be written as

$$
\begin{gathered}
A_{n}: x^{2}+c_{1} x y^{m+1}+c_{2} y^{2 m+2}+\text { (higher terms), } \quad c_{1}^{2}-4 c_{2} \neq 0, \\
D_{n+1}: x^{2} y+c_{1} x y^{m+1}+c_{2} y^{2 m+1}+\text { (higher terms), } \quad c_{1}^{2}-4 c_{2} \neq 0 .
\end{gathered}
$$

In particular, $c_{2}$ can be zero if $c_{1} \neq 0$. Note also that for $q=2 m, C_{p, q}$ can be defined by $x^{p}+x^{2} y^{2}+c_{1} x y^{m-1}+c_{2} y^{2 m}=0$ with $c_{1}^{2}-4 c_{2} \neq 0$. The notations $s B_{p, q}, s C_{p, q}, s=x, y, x y$, do not imply that $f$ is divisible by $s$ but they are just topological equivalences. So, for example $x B_{3,4}$ can be defined by $x^{4}+x y^{4}+$ (higher terms). 
2.3. Inequalities. Let $(C, P)$ be a germ of a plane curve $C$ at a singular point $P$. Denote by $\mu(C, P)$ the Milnor number of the singularity of $C$ at $P$, by $r(C, P)$ the number of locally irreducible components of $C$ at $P$, and by $\delta(C, P)$ the $\delta$-invariant, which is the maximal number of nodes in a deformation of the germ $(C, P)$. It is well-known that the equality $2 \delta(C, P)=\mu(C, P)+r(C, P)-1$ holds, see $[\mathrm{M}]$. We denote the multiplicity of $C$ at $P \in C$ by $m(C, P)$. In the case where two plane curves (or two germs) $C_{1}$ and $C_{2}$ intersect at $P$, we denote their local intersection number at $P$ by $I\left(C_{1}, C_{2} ; P\right)$.

Now we introduce several inequalities which we will use later for the classification of local singularities on reduced sextics.

Let $C$ be an irreducible plane curve in $\mathbf{P}^{2}$. We denote by $\Sigma(C)$ the set of singular points of $C$. The genus formula is

$$
g=\frac{(d-1)(d-2)}{2}-\sum_{P \in \Sigma(C)} \delta(C, P),
$$

where $g$ is the genus of a non-singular model of $C$ and $d$ is the degree of $C$. Let $P \in C$ be a singular point. The above equality and $g \geq 0$ give the inequality:

$$
\delta(C, P) \leq \frac{(d-1)(d-2)}{2}, \quad \text { or } \quad \mu(C, P)+r(C, P)-1 \leq(d-1)(d-2),
$$

called Plücker's inequality. In particular, for an irreducible sextic we have $\delta(C, P) \leq 10$.

Let $(u, v)$ be a local analytic coordinate system centered at a singular point $P \in C$. Another inequality due to Kouchnirenko $[\mathrm{K}]$ is

$$
v\left(\Gamma_{-}(f ;(u, v))\right) \leq \mu(C, P) .
$$

The last inequality, which can be applied to any reduced sextic, is $\mu(C, P) \leq 25$. This follows from the fact that the sum of Milnor numbers of a sextic function $f: \mathbf{C}^{2} \rightarrow \mathbf{C}$ is bounded above by 25 .

\section{Classification of local singularities on reduced sextics}

We give the classification of topological types of local singularities of reduced sextics for each multiplicity fixed. Suppose that a reduced sextic $C$ has an isolated singularity at the origin $O$ and let $m(C, O)$ denote its multiplicity. Since $C$ is a sextic, $m(C, O)$ can be $2,3, \ldots, 6$.

THEOREM 3. The classification of topological types of local singularities on reduced sextics is given as follows. Here $T_{0} C$ represents the tangent cone of $C$ at $O$ :

1. $m(C, O)=2$ :

(i) $T_{0} C$ is $x^{2}=0: \quad(C, O) \cong A_{n}(2 \leq n \leq 19)$.

(ii) $T_{0} C$ consists of two generic lines: $(C, O) \cong A_{1}$. 
2. $m(C, O)=3$ :

(i) $\quad T_{0} C$ is $x^{3}=0: \quad(C, O) \cong E_{n}(n=6,7,8), B_{3, n}(6 \leq n \leq 12), \quad x B_{2, n}$ $(n=5,7), C_{3, n}(7 \leq n \leq 15), D_{3, n}(10 \leq n \leq 13)$.

(ii) $T_{0} C$ is $x^{2} y=0$ : $(C, O) \cong D_{n}(5 \leq n \leq 19)$.

(iii) $T_{0} C$ consists of three generic lines: $(C, O) \cong D_{4}$.

3. $m(C, O)=4$ :

(i) $\quad T_{0} C \quad$ is $\quad x^{4}=0: \quad(C, O) \cong B_{4, n}(n=5,6), \quad D_{4,7}, \quad x B_{3, n} \quad(n=4,5)$, $S_{n}(1 \leq n \leq 6)$.

(ii) $\quad T_{0} C \quad$ is $\quad x^{3} y=0: \quad(C, O) \cong y B_{3, n}(n=4,5,6), \quad x y B_{2,3}, \quad y C_{3, n}$ $(7 \leq n \leq 12)$.

(iii) $T_{0} C$ is $x^{2} y(x+c y)=0, c \neq 0: \quad(C, O) \cong C_{4, n}(5 \leq n \leq 14)$.

(vi) $T_{0} C$ is $x^{2} y^{2}=0: \quad(C, O) \cong C_{5, n}(5 \leq n \leq 14), \quad C_{6, n}(6 \leq n \leq 12)$, $C_{7, n}(7 \leq n \leq 11), C_{8, n}(8 \leq n \leq 11), C_{9,9}$.

(v) $T_{0} C$ consists of four generic lines: $(C, O) \cong B_{4,4}$.

4. $m(C, O)=5$ :

(i) $\quad T_{0} C$ is $x^{5}=0: \quad(C, O) \cong B_{5,6}, x B_{4,5}$.

(ii) $T_{0} C$ is $x^{4} y=0: \quad(C, O) \cong y B_{4,5}, x y B_{3,4}$.

(iii) $T_{0} C$ is $x^{2} y^{3}=0: \quad(C, O) \cong y C_{5, n}(n=5,6), D_{6, n}(n=6,7)$.

(iv) $T_{0} C$ is $y^{3}\left(x+c_{1} y\right)\left(x+c_{2} y\right)=0, c_{1}, c_{2} \neq 0, c_{1} \neq c_{2}$ : $(C, O) \cong D_{6,5}$, $y C_{5,4}$.

(v) $T_{0} C$ is $x^{2} y\left(x+c_{1} y\right)\left(x+c_{2} y\right)=0, c_{1}, c_{2} \neq 0, c_{1} \neq c_{2}: \quad(C, O) \cong D_{5, n}$ $(n=6,7)$,

(vi) $T_{0} C$ is $x^{2} y^{2}(x+c y)=0, c \neq 0: \quad(C, O) \cong F_{p, q}(p, q \in\{6,7\})$.

(vii) $T_{0} C$ consists of five generic lines: $(C, O) \cong B_{5,5}$.

5. $m(C, O)=6$ :

(v) $T_{0} C$ consists of six generic lines: $(C, O) \cong B_{6,6}$.

3.1. Listing possible local singularities. Let $C$ be a reduced sextic with an isolated singularity at the origin $O$ and let $f$ be the defining polynomial of $C$ which has the form

$$
f(x, y):=\sum_{i+j \leq 6} a_{i j} x^{i} y^{j},
$$

where $a_{00}=0$. Now we make a list of possible singularities for each fixed multiplicity.

(I) Case $m(C, O)=2$ : In this case by a linear coordinate change if necessary we may assume that $a_{10}=a_{01}=0, a_{20} \neq 0$, and either $4 a_{02} a_{20}-a_{11}^{2} \neq 0$ or $a_{02}=a_{11}=0$. We look at the face $\Delta$ which has $(2,0)$ as a vertex. If $f$ is nondegenerate on $\Delta$ then $f_{\Delta}$ gives a normal form of an $A_{n}$ singularity. Otherwise, $f_{\Delta}(x, y)=a_{20}\left(x+\alpha y^{k}\right)^{2}$ for some $k \geq 2$ and $\alpha \in \mathbf{C}^{*}:=\mathbf{C}-\{0\}$. Then we take a triangular change of coordinates $x_{1}=x+\alpha y^{k}, y_{1}=y$. This operation stops at a finite number of steps and only the singularities $A_{n}, n \geq 1$, appear in this series. It is well-known that the maximal rank of the simple singularities on sextics is 19 (see [H, S-I]). Thus $n \leq 19$.

(II) Case $m(C, O)=3$ : In this case $a_{i j}=0$ for $i+j \leq 2$, and at least one 
of $a_{i j}, i+j=3$, is not zero. Put $Q={ }^{t}(1,1)$. We separate this case into three subcases according to the degeneration of the homogeneous polynomial $f_{Q}:=$ $\sum_{i+j=3} a_{i j} x^{i} y^{j}$.

(II-1) Assume that $f_{Q}$ has three distinct roots. Then the singularity is $D_{4}=B_{3,3}$.

(II-2) Assume that $f_{Q}$ has two distinct roots, one of which is a root of multiplicity 2: After a suitable linear coordinate change, we can assume that $a_{03}=$ $a_{12}=0$ and $a_{21}=1$. Thus $f(x, y)=a_{30} x^{3}+a_{21} x^{2} y+$ (higher terms). Hence the possible singularities are $D_{n}, n \geq 5$. We also have $n \leq 19$ ([H, S-I $]$ ).

(II-3) Assume that $f_{Q}$ has a root of multiplicity 3. After a suitable linear coordinate change, we can assume that $a_{03}=a_{12}=a_{21}=0$ and $a_{30}=1$. If $a_{04} \neq 0$ then the singularity is $E_{6}$. If $a_{04}=0$ and $a_{13} \neq 0$ then it is $E_{7}$. If $a_{04}=a_{13}=0$ and $a_{05} \neq 0$ then it is $E_{8}$. Otherwise $f$ is a linear combination of monomials $x^{i} y^{j}$ with $2 i+j \geq 6$.

Set $Q^{\prime}={ }^{t}(2,1)$. We again separate this case into three subcases according to the degeneration of the weighted homogeneous polynomial $f_{Q^{\prime}}$.

(II-3-1) Assume that $f_{Q^{\prime}}$ has three distinct roots. Then the singularity is $B_{3,6}$.

(II-3-2) Assume that $f_{Q^{\prime}}$ has two distinct roots, one of which is a double root. Then by a triangular change of coordinates $x_{1}=x+\alpha y^{2}, y_{1}=y$, we may assume that $f\left(x_{1}, y_{1}\right)=x_{1}^{2}\left(x_{1}+\beta y_{1}^{2}\right)+\sum^{\prime}$ where $\beta \neq 0$ and $\sum^{\prime}$ is a linear combination of monomials $x_{1}^{i} y_{1}^{j}$ with $2 i+j>6$. Now we look at the face $\Delta$ which has $(2,2)$ as the right side end and we try to make $f$ to be non-degenerate on $\Delta$. After a finite number of triangular changes of coordinates, we arrive at the form

$$
N P P(f)\left(x_{i}, y_{i}\right)= \begin{cases}x_{i}^{3}+\beta x_{i}^{2} y_{i}^{2}+c_{2} y_{i}^{n}, & c_{2} \neq 0, n: \text { odd } \\ x_{i}^{3}+\beta y_{i}^{2}\left(x_{i}^{2}+c_{1} x_{i} y_{i}^{n / 2-1}+c_{2} y^{n-2}\right), & c_{1}^{2}-4 c_{2} \neq 0, n: \text { even } .\end{cases}
$$

The corresponding singularity is $C_{3, n}, n \geq 7$. We will see later that $C_{3, n}$ with $n \leq 15$ appears but no further singularities in this series.

(II-3-3) Assume that $f_{Q^{\prime}}$ has a root of multiplicity 3. Then by a triangular change of coordinates $x_{1}=x+\alpha y^{2}, y_{1}=y$, we can write $f\left(x_{1}, y_{1}\right)=x_{1}^{3}+\sum^{\prime}$ where $\sum^{\prime}$ is a linear combination of monomials $x_{1}^{i} y_{1}^{j}$ with $2 i+j>6$. Now we look at the face $\Delta$ which contains the vertex $(3,0)$ at the right side end. We continue triangular changes of coordinates $\left(x_{i}, y_{i}\right)$ until the face function $f_{\Delta}\left(x_{i}, y_{i}\right)$ (with respect to new $\Delta$ ) becomes non-degenerate. Assume that $\left(x_{j}, y_{j}\right)$ are the last coordinates and let $(a, b)$ be the end vertex of $\Delta$ other than $(3,0)$. Note that $a \leq 2$ and $2 a+b>6$.

If $a=0$ then $f_{\Delta}\left(x_{j}, y_{j}\right)$ is a normal form of the singularity $B_{3, b}, b \geq 7$. If $a=1$ then $f_{\Delta}\left(x_{j}, y_{j}\right)=x_{j}^{3}+c_{1} x_{j} y_{j}^{b}$, which is $x B_{2, b}$. Note that if $b$ is even then $x B_{2, b}=B_{3,3 b / 2}$. We will see later that $B_{3, b}, b \geq 13$ and $x B_{2, b}, b \geq 9$ do not appear.

Assume that $a=2$. Then $f\left(x_{j}, y_{j}\right)=x_{j}^{3}+c_{1} x_{j}^{2} y_{j}^{b}+$ (higher terms). By the assumption, $b \geq 3$. If $b=3$ then we get $D_{3, n}, n \geq 10$. We will see later that $n \leq 13$. We will also see that $b \geq 4$ does not occur in reduced sextics. 
(III) Case $m(C, O)=4$ : Set $Q={ }^{t}(1,1)$. We then separate this case into five subcases according to the degeneration of the homogeneous polynomial $f_{Q}:=\sum_{i+j=4} a_{i j} x^{i} y^{j}$.

(III-1) Assume that $f_{Q}$ has four distinct roots. Then the singularity is $B_{4,4}$.

(III-2) Assume that $f_{Q}$ has three distinct roots, one of which is a double root. Then $f(x, y)=a_{40} x^{2}\left(x+c_{1} y\right)\left(x+c_{2} y\right)+$ (higher terms) with $c_{1}, c_{2} \neq 0$ and $c_{1} \neq c_{2}$. The possible singularities are only $C_{4, n}, n \geq 5$. We will see later that $n \leq 14$.

(III-3) Assume that $f_{Q}$ has two distinct double roots. After a suitable change of coordinates, we can assume that $f_{Q}(x, y)=x^{2} y^{2}$. Consider the right face $\Delta_{1}$ which contains the vertex $(2,2)$ as the left end vertex. We continue triangular changes of coordinates of type $x_{i}=x_{i-1}, y_{i}=y_{i-1}+c_{i} x_{i-1}^{k_{i}}$ successively until we arrive at the coordinates $\left(x_{\alpha}, y_{\alpha}\right)$ in which $f$ is non-degenerate on $\Delta_{1}$. Then we consider the face $\Delta_{2}$ which has $(2,2)$ as the right end vertex. We continue triangular changes of coordinates of type $x_{j}=x_{j-1}+c_{j} y_{j-1}^{\ell_{j}}, y_{j}=y_{j-1}$ until we arrive at the coordinates $\left(x_{\beta}, y_{\beta}\right)$ in which $f$ is non-degenerate on $\Delta_{2}$. Note that in this process, the part of the Newton boundary $\Gamma\left(f ;\left(x_{j-1}, y_{j-1}\right)\right) \cap$ $\{(a, b) ; b \leq 2\}$ is unchanged. So, at the end of two series of operations, we see that the possible singularities are only $C_{p, q}, p, q \geq 5$. We will see later that $C_{5, q}$, $q \geq 15, C_{6, q}, q \geq 13, C_{7, q}, q \geq 12, C_{8, q}, q \geq 12$, and $C_{9, q}, q \geq 10$ do not appear on reduced sextics.

(III-4) Assume that $f_{Q}$ has two distinct roots, one of which is a root of multiplicity 3. We can assume that $a_{40}=a_{22}=a_{13}=a_{04}=0$ and $a_{31}=1$, and $f(x, y)=x^{3} y+$ (higher terms). We focus on the face which has $(3,1)$ as the right end and do the same inductive process as in the case $m(C, O)=3$. If any one of $a_{05}, a_{14}, a_{06}$ is non-zero, $f$ is non-degenerate in the coordinate $(x, y)$ and the corresponding singularities are $y B_{3,4}, x y B_{2,3}, y B_{3,5}$.

Assume that $a_{05}=a_{14}=a_{06}=0$. For $Q^{\prime}={ }^{t}(2,1)$, the function $f_{Q^{\prime}}$ is given by $f_{Q^{\prime}}(x, y)=x^{3} y+a_{23} x^{2} y^{3}+a_{15} x y^{5}$. As $(x, y)$ are affine coordinates, we observe that $x \mid f$ and $a_{15} \neq 0$ as otherwise $x^{2} \mid f$. If $f_{Q^{\prime}}$ is non-degenerate then the singularity $(C, O)$ is $y B_{3,6}$. If it is degenerate, we apply a triangular change of coordinates $x_{1}=x+\alpha y^{2}, y_{1}=y$ and get $f_{Q^{\prime}}\left(x_{1}, y_{1}\right)=x_{1}^{3} y_{1}-\alpha x_{1}^{2} y_{1}^{3}$. Observe that $\alpha=0$ implies that $a_{23}=a_{15}=0$ and $x^{2} \mid f$, which is impossible as $(C, O)$ is assumed to be an isolated singularity. Now we consider the face which has $(2,3)$ as the right end. After suitable triangular changes of coordinates, we can assume that the Newton boundary is non-degenerate. Let $\left(x_{\alpha}, y_{\alpha}\right)$ be the last coordinates. Let $\Delta$ denote the face which contains $(2,3)$ as the right end vertex in the coordinates $\left(x_{\alpha}, y_{\alpha}\right)$ and $(a, b)$ denote the left end vertex of $\Delta$. It is easy to observe that either $(a, b)=(0, q), q \geq 8$, or $(a, b)=(1, q), q \geq 6$. In the former case the singularity $(C, O)$ is $y C_{3, q-1}$, and in the latter case it is $y C_{3,2 q-4}$. Thus in any case, we get the singularities $y C_{3, n}, n \geq 7$. We will show later that $n \leq 12$.

(III-5) Assume that $f_{Q}$ has one root of multiplicity 4. Then we may assume that $f(x, y)=x^{4}+$ (higher terms) and the singularities are $B_{4,5}$ if $a_{05} \neq 0$, and $x B_{3,4}$ if $a_{05}=0, a_{14} \neq 0$. Otherwise (i.e., $a_{05}=a_{14}=0$ ) the polynomial is a 
linear combination of monomials $x^{i} y^{j}$ with $3 i+2 j \geq 12$. Put $Q={ }^{t}(3,2)$ and consider the face function: $f_{Q}(x, y)=x^{4}+a_{23} x^{2} y^{3}+a_{06} y^{6}$. Put $J=a_{23}^{2}-4 a_{06}$. An easy discussion shows that the singularity $(C, O)$ is one of the following:

(a) If $J \neq 0, a_{06} \neq 0$, then $(C, O)$ is $B_{4,6}$.

(b) If $a_{06}=0, a_{23} \neq 0$, then $f_{Q}(x, y)=x^{4}+a_{2,3} x^{2} y^{3}$. The possible singularity in this case with $a_{15} \neq 0$ is $D_{4,7}$, as $N P P(f)=x^{4}+a_{23} x^{2} y^{3}+a_{15} x y^{5}$. If $a_{15}=0$, we can easily see that $x^{2} \mid f$.

(c) If $a_{23}=a_{06}=0$, then $a_{15}$ can not be zero and $N P P(f)=x^{4}+a_{15} x y^{5}$. Thus $(C, O)$ is $x B_{3,5}$.

(d) Assume that $a_{06}, a_{23} \neq 0$ and $J=0$. Then $N P P(f)=\left(x^{2}+\alpha y^{3}\right)^{2}$. In this case, we have no further possible triangular change of coordinates. To see the structure of the singularity, we apply toric modifications with respect to a regular subdivision admissible for the dual Newton diagram (see [O2]) and then we can easily see that the strict transforms have only $A_{n}$-type singularities on upstairs. They correspond to the sequence of singularities $S_{n}, n=1,2, \ldots, 6$.

(IV) Case $m(C, O)=5$ : Set $Q={ }^{t}(1,1)$. We separate this case into seven subcases according to the degenerations of the homogeneous polynomial $f_{Q}(x, y)$ of degree 5 .

(IV-1) Assume that $f_{Q}$ has five distinct roots. Then the singularity is $B_{5,5}$.

(IV-2) Assume that $f_{Q}$ has four distinct roots, one of which is a root of multiplicity 2. We may assume that $x^{2} \mid f_{Q}$. Then the singularities are $D_{5,6}$ if $a_{06} \neq 0$ and $D_{5,7}$ if $a_{06}=0$ and $a_{15} \neq 0$. Otherwise we can see that $x^{2} \mid f$.

(IV-3) Assume that $f_{Q}$ has three distinct roots, two of which are of multiplicity 2. We may assume that $x^{2} \mid f_{Q}$ and $y^{2} \mid f_{Q}$, and thus $f(x, y)=a_{23} x^{2} y^{3}+$ $a_{32} x^{3} y^{2}+$ (higher terms). Then the possible singularities are $F_{6,6}, F_{6,7}$ and $F_{7,7}$.

(IV-4) Assume that $f_{Q}$ has three distinct roots, one of which has the multiplicity 3. We may assume that $f_{Q}=y^{3}\left(y+\alpha_{1} x\right)\left(y+\alpha_{2} x\right)$ where $\alpha_{1}, \alpha_{2}$ are non-zero and $\alpha_{1} \neq \alpha_{2}$. Then the face $\Delta$ which has $(2,3)$ as the left side end can not be degenerate as $f$ has degree 6 . The possible singularities are $D_{6,5}$ or $y C_{5,4}$.

(IV-5) Assume that $f_{Q}$ has two distinct roots of multiplicity 2 and 3 respectively. Then we may assume that $f_{Q}(x, y)=x^{2} y^{3}$. Then the singularities obtained are $D_{6,6}, D_{6,7}, y C_{5,5}$ and $y D_{5,6}$.

(IV-6) Assume that $f_{Q}$ has two distinct roots, one of which has multiplicity 4. We assume that $f_{Q}(x, y)=x^{4} y$. Then the singularities are $y B_{4,5}$ or $x y B_{3,4}$.

(IV-7) Assume that $f_{Q}$ has a single root of multiplicity 5. Then the singularities are $B_{5,6}$ or $x B_{4,5}$.

(V) Case $m(C, O)=6$ : The singularity which can be obtained is only $B_{6,6}$.

3.2. Non-existence. We prove the non-existences of local singularities which are mentioned in the previous arguments.

Lemma 4. $A_{n}, n \geq 20$ and $D_{n}, n \geq 20$ do not appear on reduced sextics.

This is a well-known result by Horikawa and Shioda-Inose [H, S-I]. The assertion, except for the case $A_{20}$, can also be proved as follows: For $A_{2 m}$, 
$m \geq 11$, note that this singularity is locally irreducible. Thus it must be on an irreducible component. But Plücker inequality says that it can be possible only on a curve of degree greater than 7. For $A_{2 m-1}, m \geq 11$, it can not be on an irreducible curve of degree at most 6 . If it is on the intersection of two components with degrees $d_{1}, d_{2}, d_{1}+d_{2} \leq 6$, then the intersection multiplicity must be $m$. However this is also impossible by Bézout theorem as $d_{1} d_{2} \leq 9$. For $D_{n}$, $n \geq 20$, a similar argument can be applied.

\section{Lemma 5. The singularities $C_{3, n}, n \geq 16$ do not exist on reduced sextics.}

Proof. The singularity $C_{3, n}$ has two (resp. three) locally irreducible components for $n$ odd (resp. $n$ even) and its Milnor number is $n+4$. Thus $C_{3, n}$ does not exist for $n \geq 16$ on irreducible sextics by Plücker inequality. For an $n$ odd, one component has the singularity $A_{n-3}, n \geq 16$, which is locally irreducible and can not be on a curve of degree less than 6 . We prove the non-existence of $C_{3,2 k}$ for $k \geq 8$. It has three local smooth components $L_{1}, L_{2}, L_{3}$ so that their local intersection numbers are given by

$$
I\left(L_{1}, L_{j} ; O\right)=2, \quad j=2,3 \quad \text { and } \quad I\left(L_{2}, L_{3} ; O\right)=k-1 .
$$

Under the normal form of $C_{3,2 k}$ as before, the defining equation $f_{i}(x, y)$ of $L_{i}$ is given as $f_{1}(x, y)=x+y^{2}+$ (higher terms), $f_{2}(x, y)=x+\sqrt{-1} y^{k-1}+$ (higher terms) and $f_{3}(x, y)=x-\sqrt{-1} y^{k-1}+$ (higher terms), and the intersection number $I\left(L_{i}, L_{j} ; O\right)$ is easily given by these expressions (see [O2] for example). The singularity $\left(L_{2} \cup L_{3}, O\right)$ is $A_{2 k-3}$. Thus this can not be on an irreducible component of degree less than 6 . If they are on different irreducible components, say $C_{2}$ and $C_{3}$, the pair of degrees $\left(\operatorname{deg} C_{2}, \operatorname{deg} C_{3}\right)$ is $(2,4),(4,2)$ or $(3,3)$ by Bézout theorem. In particular, the local component $L_{1}$ must be on the same component with $L_{2}$ or $L_{3}$. We assume that $L_{1}$ and $L_{2}$ are on the component $C_{2}$. The pair $(2,4)$ is impossible since an irreducible conic can not have any singularity, and the case $(4,2)$ is also since $I\left(L_{1} \cup L_{2}, L_{3} ; O\right)=k+1 \geq 9$. If the pair is $(3,3)$ then $L_{1} \cup L_{2}$ must be on a cubic. Since $\left(L_{1} \cup L_{2}, O\right)$ is an $A_{3}$ singularity on the cubic, it must consist of one line and one conic. But this contradicts to the assumption that $L_{1}$ and $L_{2}$ stay in the same component $C_{2}$.

Lemma 6. The singularities $B_{3, n}, n \geq 13$ and $x B_{2, n}, n \geq 9$ do not exist on reduced sextics.

Proof. The Milnor number of $B_{3, n}$ is $2(n-1)$. Thus $n \geq 14$ is impossible by the inequality $\mu(C, O) \leq 25$. For $n=13, B_{3,13}$ is a locally irreducible singularity. Thus it must be on an irreducible component of degree less than or equal to 6 , but this is impossible. For $x B_{2, n}$, the Milnor number is $\mu\left(x B_{2, n}\right)=$ $3 n-2$. Hence $n \geq 10$ does not occur by the same reason. Suppose $n=9$. Then $x B_{2,9}$ has two locally irreducible components, one is smooth at $O$, say $L$, and the other component has the singularity $B_{2,9}=A_{8}$. But, since $A_{8}$ can not be on a cubic, we can not have the equality $I\left(L, B_{2,9} ; O\right)=9$ by Bézout theorem. 
Lemma 7. The singularities $D_{3, n}, n \geq 14$ do not exist on reduced sextics.

Proof. The singularity $D_{3, n}$ has two (resp. three) locally irreducible components for $n$ even (resp. $n$ odd) and its Milnor number is $n+7$. Thus $D_{3, n}$ does not exist for $n \geq 13$ on irreducible sextics by Plücker inequality. For $n$ even, the sextic consists of two irreducible curves and their intersection number at the origin is 6 . Hence the pair of degrees is either $(4,2)$ or $(3,3)$. But since one component has the singularity $A_{n-4}, n \geq 14$, which is locally irreducible, and this can not be on a curve of degree less than 5 .

We finally prove the non-existence of $D_{3,2 k+1}, k \geq 7$. It has three local smooth components $L_{1}, L_{2}, L_{3}$ so that their local intersection numbers are given by

$$
I\left(L_{1}, L_{j} ; O\right)=3, \quad j=2,3 \quad \text { and } \quad I\left(L_{2}, L_{3} ; O\right)=k-1 .
$$

The singularity $\left(L_{2} \cup L_{3}, O\right)$ is $A_{2 k-3}$. Thus this can not be on an irreducible component of degree less than 5. It is also impossible for $\left(L_{2} \cup L_{3}, O\right)$ to stay on an irreducible component of degree 5 since $I\left(L_{1}, L_{2} \cup L_{3} ; O\right)=6>5 \cdot 1$ contradicts to Bézout theorem. Let $C_{i}$ be the supporting irreducible component for $L_{i}, i=2,3$. Thus by Bézout theorem, the only possibility is the case (deg, $L_{2}$, $\left.\operatorname{deg}, L_{3}\right)=(2,4)$ or $(3,3)$ and $C_{i}$ must be smooth at $O$. The former case is impossible since $I\left(L_{1} \cup L_{2}, L_{3} ; O\right)=I\left(L_{1} \cup L_{3}, L_{2} ; O\right)=k+2 \geq 9$, and the latter case is also since $\left(L_{1} \cup L_{2}, O\right)$ is an $A_{5}$ singularity and it can not be on a cubic.

Lemma 8. Let $f\left(x_{j}, y_{j}\right)$ be the defining analytic function with respect to some analytic coordinates $\left(x_{j}, y_{j}\right)$ of a singularity on a sextic. Suppose that it satisfies $f_{Q}\left(x_{j}, y_{j}\right)=x_{j}^{3}+c_{1} x_{j}^{2} y_{j}^{b}$ for $Q={ }^{t}(b, 1)$. Then $b \leq 3$.

Proof. The singularity has two or three locally irreducible components. Since $\Gamma\left(f ;\left(x_{j}, y_{j}\right)\right)$ contains $\Gamma\left(x_{j}^{3}+y_{j}^{3 b}\right)$, we have $\mu\left(f ;\left(x_{j}, y_{j}\right)\right) \geq v\left(\Gamma_{-}(f ;\right.$ $\left.\left.\left(x_{j}, y_{j}\right)\right)\right)>2(3 b-1)$. Thus $b \geq 5$ is impossible by the inequality $\mu(C, O) \leq 25$. Now we assume $b=4$. Since $\mu\left(f ;\left(x_{j}, y_{j}\right)\right)>22$, the singularity can not be on an irreducible sextic. The rest of the proof is similar to the proof of the nonexistence of $D_{3, n}$ for $n \geq 14$.

Lemma 9. The singularities $C_{4, n}, n \geq 15$ do not exist on reduced sextics.

Proof. The singularity $C_{4, n}$ has three (resp. four) locally irreducible components for $n$ odd (resp. $n$ even) and its Milnor number is $n+5$. Thus $C_{4, n}$ does not exist for $n \geq 14$ on irreducible sextics by Plücker inequality. For $n$ odd, one component has the singularity $A_{n-3}, n \geq 15$, which is locally irreducible and can not be on a curve of degree less than 6 except the case where $n=15$. Suppose $n=15$. Then there are three locally irreducible components $L_{1}, L_{2}, L_{3}$ where $L_{1}$ and $L_{2}$ are smooth while $L_{3}$ is the singularity $A_{12}$ in a quintic. Since $\left(L_{1} \cup L_{3}, O\right)$ (and also $\left.\left(L_{2} \cup L_{3}, O\right)\right)$ can not be on an irreducible quintic, $\left(L_{1} \cup L_{2}, O\right)$ must be on a line, which is impossible. 
We finally prove the non-existence of $C_{4,2 k}, k \geq 8$. It has four local smooth components $L_{1}, L_{2}, L_{3}, L_{4}$ so that their local intersection numbers are given by $I\left(L_{i}, L_{j} ; O\right)=1, i=1,2, j=3,4, \quad I\left(L_{1}, L_{2} ; O\right)=1 \quad$ and $\quad I\left(L_{3}, L_{4} ; O\right)=k-1$.

The singularity $\left(L_{3} \cup L_{4}, O\right)$ is $A_{2 k-3}$. Thus this can not be on an irreducible component of degree less than 6 . If they are on different irreducible components, the pair of their degrees is either $(4,2)$ or $(3,3)$ by Bézout theorem. Let us consider the case $(4,2)$ and suppose that $L_{4}$ stays on the conic. Since $I\left(L_{1} \cup L_{2} \cup L_{3}, L_{4} ; O\right)=k+1 \geq 9$ contradicts to Bézout theorem, the conic must contain either $L_{1}$ or $L_{2}$. However this is impossible as $I\left(L_{1} \cup L_{3}, L_{2} \cup L_{4} ; O\right)=$ $k+2 \geq 10$. The case $(3,3)$ is not possible by a similar argument.

The proofs of the following two lemmas are similar to those of the previous ones. So, we omit their proofs.

Lemma 10. The singularities $C_{5, n}, n \geq 15, C_{6, n}, n \geq 13, C_{7, n}, n \geq 12, C_{8, n}$, $n \geq 12, C_{9, n}, n \geq 10$, do not exist on reduced sextics.

Lemma 11. The singularities $y C_{3, n}, n \geq 13$ do not exist on reduced sextics.

3.3. Existence. To complete the classification of local singularities on sextics, we have to show the existence of possible singularities listed in Section 3.1 and we did it by showing explicit examples of such sextics. In the calculation for listing local singularities on sextics in Section 3.1, we have implicitly shown that any of the singularities can be degenerate into one of the followings:

$$
A_{19}, D_{19}, B_{3,12}, B_{6,6}, C_{3,15}, C_{4,14}, C_{5,14}, C_{6,12}, C_{7,11}, C_{8,11}, C_{9,9}, y C_{3,12}, D_{3,13}, S_{6} \text {. }
$$

If $(C, O)$ is one of the singularities $B_{3,12}, B_{6,6}, C_{3,15}, C_{6,12}, C_{9,9}$ and $S_{6}$ then an explicit equation is given in $[\mathrm{Ph}]$ since it must be of torus type (see Section 4 below). Thus, for proving the existence, it is enough to give explicit equations of examples for the singularities $A_{19}, D_{19}, C_{4,14}, C_{5,14}, C_{7,11}, C_{8,11}, y C_{3,12}$ and $D_{3,13}$. The equations are the following:

1. $(C, O) \cong A_{19}$ (then $C$ is irreducible):

$$
\begin{aligned}
f(x, y)= & (446-54 \sqrt{5}) y^{6}-(10+26 \sqrt{5}) \sigma^{2} x y^{5}-(192+40 \sqrt{5}) \sigma y^{5} \\
& -(100+14 \sqrt{5}) \sigma x^{2} y^{4}+(978-114 \sqrt{5}) x y^{4}+(52+4 \sqrt{5}) \sigma^{2} y^{4} \\
& +(-344+24 \sqrt{5}) x^{3} y^{3}+(32-48 \sqrt{5}) \sigma^{2} x^{2} y^{3}-(384+80 \sqrt{5}) \sigma x y^{3} \\
& +(18+14 \sqrt{5}) \sigma^{2} x^{4} y^{2}+(-8+12 \sqrt{5}) \sigma x^{3} y^{2}+(618-66 \sqrt{5}) x^{2} y^{2} \\
& +(104+8 \sqrt{5}) \sigma^{2} x y^{2}+(48+10 \sqrt{5}) \sigma x^{5} y+(-344+24 \sqrt{5}) x^{4} y \\
& +(42-22 \sqrt{5}) \sigma^{2} x^{3} y-(192+40 \sqrt{5}) \sigma x^{2} y+82 x^{6}+(5+13 \sqrt{5}) \sigma^{2} x^{5} \\
& +(92+26 \sqrt{5}) \sigma x^{4}+(86-6 \sqrt{5}) x^{3}+(52+4 \sqrt{5}) \sigma^{2} x^{2}
\end{aligned}
$$

where $\sigma:=\sqrt[3]{-2+2 \sqrt{5}}$. 
2. $(C, O) \cong D_{19}$ (then $C$ is irreducible):

$$
\begin{aligned}
f(x, y)= & -7 / 2 y^{6}-3 / 4 x y^{5}+y^{5}+19 / 8 x^{2} y^{4}-7 x y^{4}+12 x^{3} y^{3} \\
& -3 / 2 x^{2} y^{3}+2 x y^{3}+x^{4} y^{2}+3 / 4 x^{3} y^{2}-7 / 2 x^{2} y^{2}-4 x^{5} y \\
& +12 x^{4} y-3 / 4 x^{3} y+x^{2} y-10 x^{6}+x^{5}-13 / 8 x^{4}
\end{aligned}
$$

3. $(C, O) \cong C_{4,14}$ (then $C$ is a reducible curve of either a conic and a quartic or two cubics):

$$
\begin{aligned}
& f(x, y)=\left(x+y^{2}\right)\left(y^{4}+x y^{3}+x y^{2}+x^{2} y+x^{4}\right) \\
& f(x, y)=\left(y^{3}+2 x y^{2}+x y-x^{3}+2 x^{2}\right)\left(y^{3}+x y^{2}+x y-x^{3}+x^{2}\right)
\end{aligned}
$$

4. $(C, O) \cong C_{5,14}$ (then $C$ is a reducible curve of a conic and a quartic):

$$
f(x, y)=\left(x+y^{2}\right)\left(y^{4}+x y^{2}+x^{4}\right)
$$

5. $(C, O) \cong C_{7,11}$ (then $C$ is irreducible):

$$
f(x, y)=y^{6}+2 y^{4} x+x^{3} y^{3}+x^{2} y^{2}+y x^{5}+y x^{4}+1 / 4 x^{6}
$$

6. $(C, O) \cong C_{8,11}$ (then $C$ is a reducible curve of a line and a quintic):

$$
f(x, y)=y\left(y^{5}+2 x y^{3}+x^{2} y+x^{5}\right)
$$

7. $(C, O) \cong y C_{3,12}$ (then $C$ is a reducible curve of a line, a conic and a cubic):

$$
f(x, y)=x\left(x y+2 x+2 y^{2}\right)\left(x^{3}-2 x y^{2}-4 x y-4 y^{3}\right)
$$

8. $(C, O) \cong D_{3,13}$ (then $C$ is a reducible curve of a conic and a quartic):

$$
f(x, y)=\left(y^{2}-x y-x^{2}-x\right)\left(y^{4}-3 x y^{3}+3 x^{2} y^{2}-2 x y^{2}+3 x^{2} y+x^{4}-x^{3}+x^{2}\right)
$$

This completes the proof of Theorem 3 .

\section{Configuration spaces of sextics of torus type}

Recall that a sextic is called of torus type if the defining polynomial $f(x, y)$ can be written as $f(x, y)=f_{2}(x, y)^{3}+f_{3}(x, y)^{2}$, where $f_{i}$ is a polynomial of degree $i$ for $i=2,3$. Note that the classification of the possible configurations of singularities of reduced sextics of torus type has been done by Pho and Oka in [Ph, O-Ph, O3].

A sextic $f_{3}^{2}+f_{2}^{3}=0$ of torus type is called tame if its singularities are sitting only at the intersection of the conic defined by $f_{2}=0$ and the cubic $f_{3}=0$. An important property of tame sextics of torus type is that the sum of intersection numbers of the conic and cubic for all singularities is always 6 . Hence there are only 11 configurations of intersection numbers of local singularities, which are described in the first column in the following table. Each vector in the first 
column is called an i-vector. The possible configurations of local singularities are described in the other two columns. This classification is done by $\mathrm{Pho}[\mathrm{Ph}]$.

\begin{tabular}{|c|c|c|}
\hline i-vector & simple singularities & non-simple singularities \\
\hline$(1,1,1,1,1,1)$ & $\left\{6 A_{2}\right\}$ & $\emptyset$ \\
\hline$(1,1,1,1,2)$ & $\left\{4 A_{2}+A_{5}\right\},\left\{4 A_{2}+E_{6}\right\}$ & $\emptyset$ \\
\hline$(1,1,2,2)$ & $\begin{array}{c}\left\{2 A_{2}+X+Y\right\} \\
X, Y=A_{5}, E_{6}\end{array}$ & $\emptyset$ \\
\hline$(1,1,1,3)$ & $\left\{3 A_{2}+A_{8}\right\}$ & $\begin{array}{c}\left\{3 A_{2}+\sigma\right\}, \\
\sigma=B_{3,6}, C_{3,7}, C_{3,8}, C_{3,9}\end{array}$ \\
\hline$(2,2,2)$ & $\begin{array}{c}\{X+Y+Z\} \\
X, Y, Z=A_{5}, E_{6}\end{array}$ & $\emptyset$ \\
\hline$(1,2,3)$ & $\begin{array}{c}\left\{A_{2}+X+A_{8}\right\} \\
X=A_{5}, E_{6}\end{array}$ & $\begin{array}{c}\left\{A_{2}, X, \tau\right\}, X=A_{5}, E_{6} \\
\tau=B_{3,6}, C_{3,7}, C_{3,8}\end{array}$ \\
\hline$(1,1,4)$ & $\left\{2 A_{2}+A_{11}\right\}$ & $\begin{array}{c}\left\{2 A_{2}+\eta\right\}, \\
\eta=C_{3,9}, B_{3,8}, C_{6,6}, B_{4,6}, D_{4,7}\end{array}$ \\
\hline$(3,3)$ & $\left\{2 A_{8}\right\}$ & $\begin{array}{c}\left\{A_{8}+\xi\right\}, \\
\xi=B_{3,6}, C_{3,7}, C_{3,8},\left\{2 B_{3,6}\right\}\end{array}$ \\
\hline$(2,4)$ & $\left\{X+A_{11}\right\}, X=A_{5}, E_{6}$ & $\begin{array}{c}\{X+\alpha\}, X=A_{5}, E_{6}, \alpha=C_{3,9}, B_{3,8} \\
\left\{A_{5}, \beta\right\}, \beta=C_{6,6}, B_{4,6}, D_{4,7}\end{array}$ \\
\hline$(1,5)$ & $\left\{A_{2}+A_{14}\right\}$ & $\left\{A_{2}+\gamma\right\}, \gamma=C_{3,12}, B_{3,10}, C_{6,9}, S_{3}$ \\
\hline$(6)$ & $\left\{A_{17}\right\}$ & $\begin{array}{c}\left\{C_{3,15}\right\},\left\{C_{9,9}\right\},\left\{B_{3,12}\right\} \\
\quad\left\{C_{6,12}\right\},\left\{S_{6}\right\},\left\{B_{6,6}\right\}\end{array}$ \\
\hline
\end{tabular}

Let $\mathscr{M}$ denote the vector space of dimension 28 , which is the space of sextics, and denote the space of sextics with a given configuration $\Sigma$ of singularities by $\mathscr{M}(\Sigma)$, i.e. $\mathscr{M}(\Sigma):=\{f \in \mathscr{M} \mid \Sigma \subseteq \Sigma(C(f))\}$, where $\Sigma(C(f))$ is the configuration of singularities of the sextic $C(f):=\left\{(x, y) \in C^{2} \mid f(x, y)=0\right\}$. On the other hand the space of sextics of torus type is of 16 dimensional, denoted by $\mathscr{T}$, and we set $\mathscr{M}_{\text {torus }}(\Sigma):=\{f \in \mathscr{T} \mid \Sigma \subseteq \Sigma(C(f))\}$ for a given configuration $\Sigma$.

The main result in this section is the following.

THEOREM 12. Let $\Sigma$ be the configuration of singularities on a tame sextic of torus type with at least one non-simple singularity (thus $\Sigma$ is one of the right side configurations in the above table). Then $\mathscr{M}(\Sigma)=\mathscr{M}_{\text {torus }}(\Sigma)$.

To prove this theorem, we study slices of the configuration spaces $\mathscr{M}(\Sigma)$ and $\mathscr{M}_{\text {torus }}(\Sigma)$. 
Proof. For each configuration $\Sigma$ in the assertion, we consider a slice condition $\mathscr{S}$ and compute the dimension of the slices of the configuration spaces $\mathscr{M}(\Sigma, \mathscr{S})$ and $\mathscr{M}_{\text {torus }}(\Sigma, \mathscr{S})$. Since obviously we have $\mathscr{M}(\Sigma, \mathscr{S}) \supset \mathscr{M}_{\text {torus }}(\Sigma, \mathscr{S})$, it is enough to show that $\mathscr{M}(\Sigma, \mathscr{S})$ is irreducible and has the same dimension as that of $\mathscr{M}_{\text {torus }}(\Sigma, \mathscr{S})$. Note that, since the set $\{f \in \mathscr{T} \mid \Sigma=\Sigma(C(f))\}$ is dense in $\mathscr{M}_{\text {torus }}(\Sigma)$ in the sense of Zariski topology, the dimension of $\mathscr{M}_{\text {torus }}(\Sigma, \mathscr{S})$ can be determined by using formulae in [O-Ph].

The proof is computational. We first start from the generic form of sextics and put the singular points at explicit loci so that we get a normal form of $\mathscr{M}(\Sigma, \mathscr{S})$. Then we check the dimension, which is the number of parameters in the normal form, and also check the irreducibility of the parameter space.

As an example, we give a proof of the assertion for the configuration $\Sigma=$ $\left\{3 A_{2}+B_{3,6}\right\}$. The dimension of a minimal slice of $\mathscr{M}_{\text {torus }}(\Sigma)$ is 4 . Let $C$ denote a sextic with this configuration. By the action of $\operatorname{PGL}(3, \mathbf{C})$, we can assume that the singularity at the origin $O$ is $B_{3,6}$, and either

(1) $\mathscr{S}_{1}$ : three $A_{2}$ 's are at $(1,0),(0,1)$ and $(1,1)$,

(2) $\mathscr{S}_{2}$ : three $A_{2}$ 's are at $(1,1),(0,1)$ and $(-1,1)$, or

(3) $B_{3,6}$ and two $A_{2}$ 's stay on a line (i.e., colinear).

We first show that case (3) does not happen. Let $L$ be the colinear line. By Bézout theorem, $L$ must be a component of $C$. However this is impossible since $A_{2}$ is locally irreducible.

Now we consider the slices $\mathscr{M}\left(\Sigma, \mathscr{S}_{i}\right), i=1,2$ :

$$
\mathscr{M}\left(\Sigma, \mathscr{S}_{i}\right)=\left\{C \in \mathscr{M}(\Sigma) \mid \Sigma(C) \text { satisfies } \mathscr{S}_{i}\right\} .
$$

In either case, the line defined by the tangent cone of $C$ at $O$ can not pass through any $A_{2}$. This can be proved as follows: Assume that $L:=\{x=0\}$ is the tangent cone of $(C, O)$ for example. If $C$ is irreducible then this contradicts Bézout theorem. We suppose that $C=L \cup C_{5}$, with $C_{5}$ is a quintic. Again this gives a contradiction that $5=I\left(L, C_{5}\right) \geq 4+2$. Thus we assume that the tangent cone of $C$ at $O$ is given by the equation $y-t x=0$ for some $t \in \mathbf{C}$. Note $t \neq 0,1$ in (1) and $t \neq \pm 1$ in (2).

We first study case (1). We start from the generic form $f(x, y)$ of sextics. Let $z_{1}:=(1,0), z_{2}:=(0,1)$ and $z_{3}:=(1,1)$ denote the singular point with $A_{2}$ singularities and get the equations

$$
f\left(z_{i}\right)=\frac{\partial f}{\partial x}\left(z_{i}\right)=\frac{\partial f}{\partial y}\left(z_{i}\right)=0, \quad \frac{\partial^{2} f}{\partial x^{2}} \frac{\partial^{2} f}{\partial y^{2}}-\frac{\partial^{2} f}{\partial x \partial y}\left(z_{i}\right)^{2}=0, \quad i=1,2,3 .
$$

We can assume that $a_{30}=1$ by the action of $\operatorname{PSL}(2, \mathbf{C})$. Next, the condition that $(C, O)$ is $B_{3,6}$ can be applied by following the same process as what we did in Section 3.1. The obtained normal form has four variables $\left(a_{14}, a_{31}, a_{40}, t\right)$ in our calculation (cf. the torus decomposition in Remark 13), and this implies that the slice is rational and thus irreducible. Thus, we can conclude that these slices coincide.

In case (2), we can also get a normal form of a minimal slice of the configuration space $\mathscr{M}(\Sigma)$ by computer calculation. Since it has four variables 
$\left(a_{42}, a_{51}, a_{60}, t\right)$ in our calculation (cf. Remark 13) and it is irreducible, we can conclude that this slice coincides with that of $\mathscr{M}_{\text {torus }}(\Sigma)$. This completes the proof for the configuration $\Sigma=\left\{3 A_{2}+B_{3,6}\right\}$.

The proofs for other configurations are analogous, so we omit them.

Remark 13. A torus decomposition of the normal form of the slice in case (1) is given by

and

$$
f_{2}(x, y)=t y^{2}-t y-x^{2}+x
$$

$$
\begin{aligned}
f_{3}(x, y)= & \left(\left(2 a_{40}+6\right) x^{3}+\left(3 t-a_{41}-a_{31}\right) x^{2} y-\left(2 a_{40}+6\right) x^{2}\right. \\
& +\left(-9 t+2 a_{31}+a_{41}\right) x y^{2}+\left(6 t-a_{31}\right) x y-\left(2 a_{40} t+a_{31}\right) t y^{3} \\
& \left.+\left(2 a_{40} t+a_{31}\right) t y^{2}\right) /\left(2 \sqrt{3+a_{40}}\right) .
\end{aligned}
$$

The torus decomposition in case (2) is

$$
f_{2}(x, y)=t y^{2}-t y-x y+x
$$

and

$$
\begin{aligned}
f_{3}(x, y)= & \left(-8 a_{60}^{2} x^{3}-4 a_{60} a_{51} x^{2} y+4 a_{60} a_{51} x^{2}+\left(a_{51}^{2}-4 a_{60} a_{42}\right) x y^{2}\right. \\
& +\left(8 a_{60}^{2}+4 a_{60} a_{42}-a_{51}^{2}\right) x y+\left(4 a_{60} a_{51} t+4 a_{60} a_{42}+8 a_{60}^{2}-a_{51}^{2}\right) t y^{3} \\
& \left.+\left(-4 a_{60} a_{51} t+a_{51}^{2}-8 a_{60}^{2}-4 a_{60} a_{42}\right) t y^{2}\right) /\left(8 a_{60}^{3 / 2}\right) .
\end{aligned}
$$

Remark 14. For any given configuration $\Sigma$ of the singularities of a tame sextic of torus type with only simple singularities (thus $\Sigma$ is one of the left side configurations in the table), there exists a sextic of non-torus type with the same configuration $\Sigma$ of singularities $([\mathrm{O} 5])$. Thus they make a Zariski pair for which the Alexander polynomials are given by $t^{2}-t+1$ and 1 . Eyral and Oka have proved that, for 7 tame configurations $\Sigma$ 's, there exists a sextic $C_{\Sigma}$ of non-torus type with configuration $\Sigma$ so that the fundamental group $\pi_{1}\left(\mathbf{P}^{2} \backslash C_{\Sigma}\right)$ is abelian. See $[\mathrm{E}-\mathrm{O}]$ for details.

Remark 15. For $A_{17}$, we can also check the assertion of Artal: The parameter space of sextics having $A_{17}$ as a singularity consists of four connected components, two of which correspond to irreducible sextics of torus and nontorus type and the others correspond to reducible ones of torus and non-torus type.

\section{Relation between sextics of torus type and their $\rho$-invariants}

Let $C$ be a plane curve of degree $d$ defined by $f(x, y)=0$ and let $\Sigma(C)$ denote the singular locus of $C$. Let $P \in \Sigma(C)$ be a singular point on $C$. Consider an embedded resolution of $C, \pi: \tilde{U} \rightarrow U$ where $U$ is an open neighborhood of $P$, and let $E_{1}, \ldots, E_{s}$ denote the exceptional divisors. Choose a local 
coordinate system centered at $P$ and let $k_{i}$ and $m_{i}$ be the order of zero of the canonical two form $\pi^{*}(d u \wedge d v)$ and $\pi^{*} f$ respectively along the divisor $E_{i}$. Then we define an ideal $\mathscr{J}_{P, k, d}$ and integers $\rho(P ; k)$ and $\rho(k)$ by

$$
\mathscr{J}_{P, k, d}:=\left\{\phi \in \mathcal{O}_{P} ;\left(\pi^{*} \phi\right) \geq \sum_{i}\left(-k_{i}+\left[k m_{i} / d\right]\right) E_{i}\right\}
$$

and

$$
\rho(P, k):=\operatorname{dim} \mathcal{O}_{P} / \mathscr{J}_{P, k, d}, \quad \rho(k)=\sum_{P \in \Sigma(C)} \rho(P, k)
$$

respectively. These notations are used in [A] and [O4].

By easy calculation we can obtain the generators of the ideals $\mathscr{J}_{P, k, 6}$ and the $\rho(P, k)$ for all non-simple singularities in sextics $d=6$. Note that the $\rho$ invariants of simple singularities in sextics are aligned in [O4, Proposition 3]. The following is the list of $\rho$-invariants of non-simple singularities appearing on reduced sextics. Here the notation $\rho_{3,5}(P)$ represents the triple $(\rho(P, 5), \rho(P, 4)$, $\rho(P, 3))$ :

1. $B_{4,4}, C_{4,5}, C_{5,5}: \mathscr{J}_{P, k, 6}=\left\langle x^{2}, x y, y^{2}\right\rangle,\langle x, y\rangle,\langle x, y\rangle$ and $\rho_{3,5}(P)=(3,1,1)$.

2. $B_{4,5}, y B_{3,4}: \mathscr{J}_{P, k, 6}=\left\langle x^{2}, x y, y^{2}\right\rangle,\left\langle x, y^{2}\right\rangle,\langle x, y\rangle$ and $\rho_{3,5}(P)=(3,2,1)$.

3. $B_{3,6}, B_{3,7}, y B_{3,5}, y B_{3,6}, y B_{4,3}, y B_{5,2}, x y B_{2,3}, C_{3,7}, C_{3,8}, C_{4,6}, C_{4,7}, C_{4,8}, C_{5,6}$, $C_{5,7}, C_{5,8}: \mathscr{J}_{P, k, 6}=\left\langle x^{2}, x y, y^{3}\right\rangle,\left\langle x, y^{2}\right\rangle,\langle x, y\rangle$ and $\rho_{3,5}(P)=(4,2,1)$.

4. $B_{3,8}, C_{3,9}, C_{3,10}, C_{3,11}, C_{4,9}, C_{4,10}, C_{4,11}, C_{5,9}, C_{5,10}, C_{5,11}, y C_{3,7}$ : $\mathscr{J}_{P, k, 6}=\left\langle x^{2}, x y, y^{4}\right\rangle,\left\langle x, y^{2}\right\rangle,\langle x, y\rangle$ and $\rho_{3,5}(P)=(5,2,1)$.

5. $B_{3,9}, y C_{3,8}, y C_{3,9}, D_{3,10}: \mathscr{J}_{P, k, 6}=\left\langle x^{2}, x y, y^{4}\right\rangle,\left\langle x, y^{3}\right\rangle,\langle x, y\rangle$ and $\rho_{3,5}(P)=(5,3,1)$.

6. $B_{4,6}, y B_{5,3}, D_{4,7}: \mathscr{J}_{P, k, 6}=\left\langle x^{2}, x y^{2}, y^{3}\right\rangle,\left\langle x^{2}, x y, y^{2}\right\rangle,\langle x, y\rangle$ and $\rho_{3,5}(P)=(5,3,1)$.

7. $C_{6,6}, C_{6,7}, C_{6,8}, C_{7,7}, C_{7,8}, C_{8,8}$ : $\mathscr{J}_{P, k, 6}=\left\langle x^{3}, x y, y^{3}\right\rangle,\left\langle x^{2}, x y, y^{2}\right\rangle,\langle x, y\rangle$ and $\rho_{3,5}(P)=(5,3,1)$.

8. $S_{1}, S_{2}: \mathscr{J}_{P, k, 6}=\left\langle x^{2}, x y^{2}, y^{3}\right\rangle,\left\langle x^{2}, x y, y^{2}\right\rangle,\langle x, y\rangle$ and $\rho_{3,5}(P)=(5,3,1)$.

9. $B_{3,10}, B_{3,11}, y B_{7,2}, C_{3,12}, C_{3,13}, C_{3,14}, C_{4,12}, C_{4,13}, C_{4,14}, C_{5,12}, C_{5,13}, C_{5,14}$, $y C_{3,10}, y C_{3,11}, y C_{3,12}, D_{3,11}, D_{3,12}, D_{3,13}$ : $\mathscr{J}_{P, k, 6}=\left\langle x^{2}, x y, y^{5}\right\rangle,\left\langle x, y^{3}\right\rangle,\langle x, y\rangle$ and $\rho_{3,5}(P)=(6,3,1)$.

10. $B_{5,5}, B_{5,6}, y B_{4,5}, y B_{5,4}, x y B_{3,4}, y C_{5,4}, y C_{5,5}, y C_{5,6}, D_{5,6}, D_{5,7}, D_{6,5}, D_{6,6}$, $D_{6,7}, F_{6,6}, F_{6,7}, F_{7,7}$ : $\mathscr{J}_{P, k, 6}=\left\langle x^{3}, x^{2} y, x y^{2}, y^{3}\right\rangle,\left\langle x^{2}, x y, y^{2}\right\rangle,\langle x, y\rangle$ and $\rho_{3,5}(P)=(6,3,1)$.

11. $C_{6,9}, C_{6,10}, C_{6,11}, C_{7,9}, C_{7,10}, C_{7,11}, C_{8,9}, C_{8,10}, C_{8,11}$ : $\mathscr{J}_{P, k, 6}=\left\langle x^{3}, x y, y^{4}\right\rangle,\left\langle x^{2}, x y, y^{2}\right\rangle,\langle x, y\rangle$ and $\rho_{3,5}(P)=(6,3,1)$.

12. $S_{3}, S_{4}, S_{5}: \mathscr{J}_{P, k, 6}=\left\langle x^{3}, x y^{2}, x^{2}-y^{3}, y^{4}\right\rangle,\left\langle x^{2}, x y, y^{2}\right\rangle,\langle x, y\rangle$ and $\rho_{3,5}(P)=(6,3,1)$.

13. $C_{3,15}: \mathscr{J}_{P, k, 6}=\left\langle x^{2}, x y, y^{6}\right\rangle,\left\langle x, y^{3}\right\rangle,\langle x, y\rangle$ and $\rho_{3,5}(P)=(7,3,1)$.

14. $C_{9,9}: \mathscr{J}_{P, k, 6}=\left\langle x^{4}, x y, y^{4}\right\rangle,\left\langle x^{2}, x y, y^{2}\right\rangle,\langle x, y\rangle$ and $\rho_{3,5}(P)=(7,3,1)$.

15. $C_{6,12}: \mathscr{J}_{P, k, 6}=\left\langle x^{3}, x y, y^{5}\right\rangle,\left\langle x^{2}, x y, y^{3}\right\rangle,\langle x, y\rangle$ and $\rho_{3,5}(P)=(7,4,1)$. 
16. $S_{6}: \mathscr{J}_{P, k, 6}=\left\langle x^{3}, x^{2} y, x^{2}-y^{3}, y^{4}\right\rangle,\left\langle x^{2}, x y, y^{3}\right\rangle,\langle x, y\rangle$ and $\rho_{3,5}(P)=(7,4,1)$.

17. $B_{3,12}: \mathscr{J}_{P, k, 6}=\left\langle x^{2}, x y^{2}, y^{6}\right\rangle,\left\langle x, y^{4}\right\rangle,\left\langle x, y^{2}\right\rangle$ and $\rho_{3,5}(P)=(8,4,2)$.

18. $B_{6,6}: \mathscr{J}_{P, k, 6}=\left\langle x^{4}, x^{3} y, x^{2} y^{2}, x y^{3}, y^{4}\right\rangle,\left\langle x^{3}, x^{2} y, x y^{2}, y^{3}\right\rangle,\left\langle x^{2}, x y, y^{2}\right\rangle$ and $\rho_{3,5}(P)=(10,6,3)$.

THEOREM 16. Let $C$ be a sextic with at least one non-simple singularity. If $C$ satisfies $\rho(5) \geq 7$ then it is of torus type.

The proof for the case where the non-simple singularity is a higher triple point is given in Proposition 17. Here a higher triple point means a non-simple singularity with multiplicity 3 . The proof for the case where the non-simple singularity is a quadruple point is given in Proposition 18. The rest, i.e., the case where it has multiplicity 5 or 6 , will be dealt with after these propositions.

Proposition 17. Let $C$ be a sextic with a higher triple point. If $C$ satisfies $\rho(5) \geq 7$ then it is of torus type.

Proof. We briefly describe a method as used in [W] to read off all possible configurations of singularities on sextics with at least a higher triple point. Let $C$ be given by

$$
F(x, y, z)=x^{3} z^{3}+x^{2} z^{2} a(y, z)+x z b(y, z)+c(y, z)=0,
$$

where $a, b$ and $c$ respectively are homogeneous polynomials of degree 2, 4 and 6 . The point $P=(1: 0: 0)$ is a higher triple point. Let $X$ be the double covering of $\boldsymbol{P}^{2}$ determined by $C$. Then $X$ is an elliptic surface defined by $w^{2}=F$ in the weighted projective space $\boldsymbol{P}(3,1,1,1)$.

By [W, Theorem 5.1], the surface $X$ is not rational if and only if $\Sigma(C)=$ $\left\{2 B_{3,6}\right\}$ or $\Sigma(C)=\left\{B_{3,12}\right\}$. Both are of torus type.

Suppose that $X$ is a rational elliptic surface. Then the configuration of exceptional fibres of $X$ determines the singularities on $C$ as describe in the following table, see $[\mathrm{W}$, Section 5] for details. Here $\rho(-, 5)$ represents the $\rho$-invariants of the singularities described in the second column. Note that $C_{3,6}=B_{3,6}$ and $D_{3,9}=B_{3,9}$.

\begin{tabular}{|c|c|c|c|c|}
\hline Type & Singularity & $\rho(-, 5)$ & Singularity at $P$ & $\rho(P, 5)$ \\
\hline$I_{r}$ & $A_{r-1}$ & $\leq 3$ & $C_{3,6+r}$ & $4, \ldots, 7$ \\
$I I$ & - & - & $B_{3,7}$ & 4 \\
$I I I$ & $A_{1}$ & 0 & $y B_{5,2}$ & 4 \\
$I V$ & $A_{2}$ & 1 & $B_{3,8}$ & 5 \\
$I_{r}^{*}$ & $D_{4+r}$ & $\leq 2$ & $D_{3,9+r}$ & 5 or 6 \\
$I V^{*}$ & $E_{6}$ & 2 & $B_{3,10}$ & 6 \\
$I I I^{*}$ & $E_{7}$ & 2 & $y B_{7,2}$ & 6 \\
$I I^{*}$ & $E_{8}$ & 2 & $B_{3,11}$ & 6 \\
\hline
\end{tabular}


Let $d$ be an exceptional fibre. If the fibre $d$ is different from the fibre $z=0$ then $C$ has another singularity on $d \cap C$ of the same type as $X$ has on the fibre and this singularity is given in the second column. If the fibre $d$ is $z=0$ then $C$ has $P$ as the unique singularity on $d \cap C$. The type of singularity at $P$ is given in the fourth column except for the following cases:

- If $z$ divides $c$ but $z$ does not divide $b$ then the fibre $z=0$ has the singularity of type $I_{r}$ for $r \geq 0$ and $C$ has another $A_{1}$ singularity on $z=0$.

- If $z$ divides both $b$ and $c$ but does not divide $a$, then the fibre $z=0$ has type $I_{1}$ and the singularity at $P$ is of type $C_{3,8}$.

- If $z$ divides all $a, b$ and $c$ then the fibre $z=0$ has type $I I$ and the singularity at $P$ is of type $y B_{5,2}$.

From the list of $\rho$-invariants of non-simple singularities and the fact that $B_{3,12}$ corresponds only to a non-rational surface, we can see that the possible values of $\rho(P, 5)$ for a higher triple point $P$ are $4 \leq \rho(P, 5) \leq 7$. We now check the assertion case by case.

(1) Case $\rho(P, 5)=7$ : The singularity at $P$ is $C_{3,15}$ and hence the corresponding fibre is $I_{9}$. From the list of all possible configurations of singularities on rational elliptic surfaces $[\mathrm{Pe}]$, we have only one case, that is $I_{9} 3 I_{1}$. The corresponding sextic has $\Sigma(C)=\left\{C_{3,15}\right\}$ or $\Sigma(C)=\left\{C_{3,15}, A_{1}\right\}$. By Theorem 12, such a sextic is of torus type.

(2) Case $\rho(P, 5)=6$ : The singularity at $P$ is $C_{3,6+r}$ for $6 \leq r \leq 8, D_{3,9+j}$ for $2 \leq j \leq 4, B_{3,10}, B_{3,11}$ or $y B_{7,2}$. This means that the fibre $z=0$ is one of

$$
\left\{I_{r}, I_{j}^{*}, I V^{*}, I I I^{*}, I I^{*} \mid 6 \leq r \leq 8,2 \leq j \leq 4\right\} .
$$

For obtaining $\rho(5) \geq 7$, the sextic should have at least one singularity other than $P$ whose $\rho$-invariant is strictly positive. From the list in $[\mathrm{Pe}]$, we see that this condition is satisfied only in the following cases: $I_{6} I V 2 I_{1}, I_{6} I_{3} I_{2} I_{1}, I_{6} I_{3} 3 I_{1}$, $V I^{*} V I, V I^{*} I_{3} I_{1}$. Then the possible configurations of singularities are $\Sigma(C)=$ $\left\{C_{3,12}, A_{2}, i A_{1}\right\}$ for $0 \leq i \leq 2$ and $\Sigma(C)=\left\{B_{3,10}, A_{2}, j A_{1}\right\}$ for $j=0,1$. From Theorem 12, all of them are of torus type.

(3) Case $\rho(P, 5)=5$ : The singularity at $P$ is $C_{3,6+r}$ for $3 \leq r \leq 5, D_{3,9+j}$ for $j=0,1$ or $B_{3,8}$. Note that $D_{3,9}=B_{3,9}$. The fibre $z=0$ is $I_{r}$ for $3 \leq r \leq 5, I_{j}^{*}$ for $j=0,1$ or $I V$. From the list in [Pe], we see that only the following cases correspond to sextics with $\rho(5) \geq 7$ :

- (the fibre $z=0$ is $I_{3}$ ): $4 I_{3}, I_{6} I_{3} I_{2} I_{1}, I_{6} I_{3} 3 I_{1}, 3 I_{3} I_{2} I_{1}, 3 I_{3} 3 I_{1}, I V 2 I_{3} 2 I_{1}$, $I V 2 I_{3} I_{2}, I V^{*} I_{3} I_{1}$. The first case gives $\Sigma(C)=\left\{C_{3,9}, 3 A_{2}, i A_{1}\right\}$ for $i=0,1$. The last one gives $\Sigma(C)=\left\{C_{3,9}, E_{6}, i A_{1}\right\}$ for $i=0,1$. The other cases give either $\Sigma(C)=\left\{C_{3,9}, 2 A_{2}, i A_{1}\right\}$ for $0 \leq i \leq 2$ or $\Sigma(C)=\left\{C_{3,9}, A_{5}, i A_{1}\right\}$ for $0 \leq i \leq 2$. All of them are of torus type.

- (the fibre $z=0$ is $I V$ ): $3 I V, I V 2 I_{3} I_{2}, I V 2 I_{3} 2 I_{1}, I_{6} I V 2 I_{1}, I V^{*} I V$. The last two cases respectively give $\Sigma(C)=\left\{B_{3,8}, A_{5}\right\}$ and $\Sigma(C)=\left\{B_{3,8}, E_{6}\right\}$. The other cases give $\Sigma(C)=\left\{B_{3,8}, 2 A_{2}, i A_{1}\right\}$ for $i=0,1$. All of them are of torus type.

(4) Case $\rho(P, 5)=4$ : The singularity at $P$ is $C_{3,6+r}$ for $0 \leq r \leq 2, B_{3,7}$ or 
$y B_{5,2}$. Then the fibre $z=0$ is $I_{r}$ for $1 \leq r \leq 2, I I, I I I$ or not exceptional. From the list in $[\mathrm{Pe}]$, we see that only the following cases correspond to sextics with $\rho(5) \geq 7$ :

- (the fibre $z=0$ is $I_{2}$ ): $3 I_{3} I_{2} I_{1}, I V 2 I_{3} I_{2}, I_{6} I_{3} I_{2} I_{1}$. The first two cases give $\Sigma(C)=\left\{C_{3,8}, 3 A_{2}, i A_{1}\right\}$ for $i=0,1$ and the last case gives $\Sigma(C)=\left\{C_{3,8}\right.$, $\left.A_{5}, A_{2}, i A_{1}\right\}$ for $i=0,1$. All of them are of torus type.

- (the fibre $z=0$ is $I_{1}$ ): $I_{9} 3 I_{1}, I_{6} I V 2 I_{1}, I_{6} I_{3} 3 I_{1}, I_{6} I_{3} I_{2} I_{1}, I V 2 I_{3} 2 I_{1}, 3 I_{3} I_{2} I_{1}$, $3 I_{3} 3 I_{1}, I V^{*} I_{3} I_{1}$. The first case gives $\Sigma(C)=\left\{C_{3,6+i}, A_{8}, j A_{1}\right\}$ for $i=1,2$ and $j=0,1$. The last case gives $\Sigma(C)=\left\{C_{3,6+i}, E_{6}, A_{2}, j A_{1}\right\}$ for $i=1,2$ and $j=0,1$. The other cases give either $\Sigma(C)=\left\{C_{3,6+i}, A_{5}, A_{2}, j A_{1}\right\}$ for $i=1,2$ and $0 \leq j \leq 2$ or $\Sigma(C)=\left\{C_{3,6+i}, 3 A_{2}, j A_{1}\right\}$ for $i=1,2$ and $0 \leq j \leq 2$. All of them are of torus type.

- (the fibre $z=0$ is not exceptional): $I_{9} 3 I_{1}, I V^{*} I V, I V^{*} I_{3} I_{1}, I_{6} I V 2 I_{1}$, $I_{6} I_{3} I_{2} I_{1}, I_{6} I_{3} 3 I_{1}, 3 I V, I V 2 I_{3} I_{2}, I V 2 I_{3} 2 I_{1}, 4 I_{3}, 3 I_{3} I_{2} I_{1}, 3 I_{3} 3 I_{1}$. The first case gives $\Sigma(C)=\left\{B_{3,6}, A_{8}, i A_{1}\right\}$ for $i=0,1$. The second and the third cases give $\Sigma(C)=\left\{B_{3,6}, E_{6}, A_{2}, i A_{1}\right\}$ for $i=0,1$. The last six cases give $\Sigma(C)=$ $\left\{B_{3,6}, 3 A_{2}, i A_{1}\right\}$ for $0 \leq i \leq 2$. The other cases give $\Sigma(C)=\left\{B_{3,6}, A_{5}, A_{2}\right.$, $\left.i A_{1}\right\}$ for $0 \leq i \leq 2$. All of them are of torus type.

Proposition 18. Let $C$ be a sextic with a quadruple point. If $C$ satisfies $\rho(5) \geq 7$ then it is of torus type.

Proof. First of all, we briefly describe an argument as used in [W] to obtain all possible configurations of sextics with a quadruple point. Suppose that $C$ is given by

$$
F(x, y, z)=x^{2} a(y, z)+2 x b(y, z)+c(y, z)=0,
$$

where $a, b$ and $c$ are homogeneous polynomials of degree 4,5 and 6 respectively. We denote $f(y, z)=F(1, y, z)$. Let $P=(1: 0: 0)$ be the singular point.

Denote $\delta=b^{2}-a c$. Let $d$ be a non-zero linear form in $\boldsymbol{C}[y, z]$. For $g \in \boldsymbol{C}[y, z]$, denote $v_{d}(g)=k$ if $g=d^{k} g_{1}$ and $d \nmid g_{1}$. The singularities of $C$ can be realized as follows, see [W, Section 1,2 and 6] for details:

(i) $v_{d}(\delta)=r \geq 2$ and $v_{d}(a) \leq 1$. The sextic $C$ has another singularity on $d \cap C$ of type $A_{r-1}$ if $v_{d}(f)=0$ and of type $D_{r}$ if $v_{d}(f)=1$ (here we mean $D_{2}=2 A_{1}$ and $\left.D_{3}=A_{3}\right)$.

(ii) $v_{d}(\delta)=2, v_{d}(a) \geq 2$ and $v_{d}(f)=1$. The sextic has only one $A_{1}$ singularity on $d \cap C$.

(iii) Other possible cases of $v_{d}(\delta)$ and $v_{d}(a)$ correspond to sextics with $P$ as the unique singular point on $C \cap d$. Moreover, the relation between the values of $v_{d}(\delta)$ and $v_{d}(a)$ and the singularity at $P$ is given in the following table. Here, for convenience, we choose $d$ in such a way that $v_{d}(a) \leq v_{l}(a)$ for any non-zero linear form $l$. 


\begin{tabular}{|c|c|c|c|c|}
\hline$v_{d}(a)$ & $v_{d}(f)$ & $v_{d}(\delta)$ & Singularity at $P$ & $\rho(P, 5)$ \\
\hline$\leq 1$ & $\leq 1$ & $\leq 10$ & $C_{4,4}=B_{4,4}$ & 3 \\
\hline 2 & 0 & 0 & $C_{4,5}$ or $C_{5,5}$ & 3 \\
\hline 2 & 0 & $s \geq 2$ & $C_{4+r, 4+s} ; r+s \leq 10$ or $(r, s)=(1,10)$ & $4, \ldots, 7$ \\
\hline 2 & 1 & 2 & $C_{4+r, 6} ; 0 \leq r \leq 8$ & $4, \ldots, 7$ \\
\hline 2 & 1 & 3 & $C_{4+r, 8} ; 0 \leq r \leq 7$ & $4, \ldots, 6$ \\
\hline 3 & 0 & 0,2 or 3 & $y B_{3,4}, x y B_{2,3}$ or $y B_{3,5}$ & 3,4 \\
\hline 3 & 1 & 2 & $x y B_{2,3}$ & 4 \\
\hline 3 & 1 & $s \geq 4$ & $y C_{3,2+s} ; 4 \leq s \leq 10$ & $4,5,6$ \\
\hline 4 & 0 & 0,2 & $B_{4,5}, y B_{4,3}$ & 3,4 \\
\hline 4 & 1 & 2 & $y B_{4,3}$ & 4 \\
\hline 4 & 0 & $k \geq 4$ & $S_{k-4} ; 4 \leq k \leq 10\left(S_{0}=B_{4,6}\right)$ & $5,6,7$ \\
\hline 4 & 1 & 4 & $D_{4,7}$ & 5 \\
\hline 4 & 1 & 5 & $y B_{5,3}$ & 5 \\
\hline
\end{tabular}

We now check the assertion by considering case by case.

(1) Case where $P$ is of type $C_{p, q}$ :

- If $\rho(P, 5)=3$ then $P$ is one of $C_{4,4}, C_{4,5}$ or $C_{5,5}$. By the above argument, we see that configurations of other singularities with positive values of $\rho(-, 5)$ which may occur in $\Sigma(C)$ are either $\left\{A_{i}\right\}$ or $\left\{D_{i+1}\right\}$ for $i \leq 9$, $\left\{m A_{j}\right\}$ or $\left\{m D_{j+1}\right\}$ for $m \leq 3$ and $j \leq 3$, or $\left\{Q_{i}, Q_{j}^{\prime}\right\}$ where $Q_{i}=A_{i}$ or $D_{i+1}$ for $i \leq 6$ and $Q_{j}^{\prime}=A_{j}$ or $D_{j+1}$ for $j \leq 3$. All of these cases give $\rho(5) \leq 6$.

- If $\rho(P, 5)=4$ then $P$ is of type $C_{4, q}$ or $C_{5, q}$ for $6 \leq q \leq 8$. We see that configurations of other singularities with positive values of $\rho(-, 5)$ may occur in $\Sigma(C)$ are either $\left\{A_{i}\right\}$ or $\left\{D_{i+1}\right\}$ for $i \leq 7,\left\{m A_{j}\right\}$ or $\left\{m D_{j+1}\right\}$ for $m \leq 2, j \leq 3$, or $\left\{Q_{2}, Q_{j}^{\prime}\right\}$ where $Q_{2}=A_{2}$ or $D_{3}$ and $Q_{j}^{\prime}=A_{j}$ or $D_{j+1}$ for $j \leq 4$. All of these cases give $\rho(5) \leq 6$.

- Suppose that $\rho(P, 5)=5$. If $P$ is of type $C_{4, q}, C_{5, q}$ for $9 \leq q \leq 11$ or $C_{6+i, q}$ for $i=1,2$ and $r=7,8$, or $C_{8,8}$ then one more singularity with positive value of $\rho(-, 5)$ which can occur in $\Sigma(C)$ is $A_{i}$ or $D_{i+1}$ for $i \leq 4$. All of them give $\rho(5) \leq 6$. If $P$ is of type $C_{6,6}$ then the configurations $\Sigma(C)=\left\{C_{6,6}, A_{5}\right\}$ and $\Sigma(C)=\left\{C_{6,6}, 2 A_{2}\right\}$ satisfy $\rho(5)=7$ and both are of torus type.

- If $\rho(P, 5)=6$ then $P$ is one of type $C_{4, q}, C_{5, q}$ for $12 \leq q \leq 14$ or $C_{6+i, q}$ for $i=0,1,2$ and $9 \leq q \leq 11$. There is only one case with $\rho(5) \geq 7$, that is $\Sigma(C)=\left\{C_{6,9}, A_{2}\right\}$. This is of torus type. 
- If $\rho(P, 5) \geq 7$ then there are two possible cases $\Sigma(C)=\left\{C_{6,12}\right\}$ and $\Sigma(C)=$ $\left\{C_{9,9}\right\}$. Both are of torus type.

(2) Case where $P$ is $B_{4,5}$ or has one of types $y B_{p, q}, x y B_{p, q}$ and $y C_{3, q}$ : Using the same argument as above, we can conclude that there is no sextic with $\rho(5) \geq 7$.

(3) Case where $P$ is of type $B_{4,6}$ or $S_{n}$ for $1 \leq n \leq 6$ : If $P=B_{4,6}$ or $S_{n}$ for $n=1,2$ then $\rho(P, 5)=5$. Then the cases with $\rho(5) \geq 7$ are $\Sigma(C)=\left\{B_{4,6}, A_{5}\right\}$ or $\Sigma(C)=\left\{B_{4,6}, 2 A_{2}\right\}$. Both are of torus type. If $P$ is one of $S_{n}$ for $n=3,4,5$ then $\rho(P, 5)=6$. Then there is only one case $\Sigma(C)=\left\{S_{3}, A_{2}\right\}$ with $\rho(5)=7$. This is also of torus type. If $P$ is $S_{6}$ then we also know that the sextic should be of torus type with $\rho(5)=7$.

(4) Case where $P$ is $D_{4,7}$ : We have $\rho(P, 5)=5$ and the cases with $\rho(5) \geq 7$ are $\Sigma(C)=\left\{D_{4,7}, A_{5}\right\}$ and $\Sigma(C)=\left\{D_{4,7}, 2 A_{2}\right\}$. Both are of torus type.

Proof of Theorem 16. If $C$ contains one non-simple singularity of multiplicity 3 or 4 then the result follows from the previous propositions. We consider the singularity with multiplicity 5 or 6 . The case of multiplicity 6 is trivial since the sextic factors into 6 distinct lines and it has only one singularity at the common point. This singularity is of type $B_{6,6}$ and has $\rho(-, 5)=10$. The sextic is of torus type.

We consider $C$ with a quintuple point $P$. There are 16 possible types of singularities for $P$ and $\rho(P, 5)=6$. Suppose that $C$ has another singular point $Q$, then Bézout inequality implies that the line $\overline{P Q}=l \subset C$. We write $C=l \cup C_{5}$. Since $I\left(l, C_{5} ; Q\right) \geq 1$ we have $I\left(l, C_{5} ; P\right) \leq 4$. Because $(C, P)$ is a quintuple point, $I\left(l, C_{5} ; P\right)=4$ and $I\left(l, C_{5} ; Q\right)=1$. Thus $(C, Q)$ is an $A_{1}$ singularity and hence $\rho(5)=6$. This completes the proof.

We conclude this paper with conjectures posed by the third author.

Conjecture A. The generic Alexander polynomial of a reduced sextic $C$ is trivial if and only if it is of non-torus type.

See $[A$, O4] for the definition of generic Alexander polynomials.

CONJECTURe $\mathrm{A}^{\prime}$. If a reduced sextic satisfies $\rho(5) \geq 7$ then it is of torus type.

Note that Theorem 16 verifies Conjecture $\mathrm{A}^{\prime}$ for the case where a sextic has at least one non-simple singularity.

Conjecture B. Let $C$ be an irreducible sextic of non-torus type. Then the fundamental group $\pi_{1}\left(\mathbf{P}^{2}-C\right)$ is isomorphic to $\mathbf{Z} / 6 \mathbf{Z}$.

We remark that there is a reducible sextic $C$ of non-torus type whose fundamental group $\pi_{1}\left(\mathbf{P}^{2}-C\right)$ is not abelian. 
Acknowledgements. The computation in this paper has been done by using the SCURVE program made by Pho Duc Tai and the authors would like to express deep thanks to him. The second author would like to thank Department of Mathematics, Tokyo Metropolitan University for excellent facilities and marvelous hospitality, to Japan Society for the Promotion of Science for financial support.

\section{REFERENCES}

[A] E. Artal, Sur les couples des Zariski, J. Algebraic Geometry 3 (1994), 223-247.

[AGV] V. I. Arnol'd, A. N. Varchenko, S. M. Gusein-Zade, Singularities of differentiable mappings, Vol. 1, Birkhäuser, 1985.

[D] A. I. Degtyarev, Classification of surfaces of degree four having a non-simple singular point, Math. USSR Izvestiya 35 (1990), 607-627.

[E-O] C. Eyral, M. OKa, On the fundamental groups of the complements of plane singular sextics, preprint.

[H] E. Horikawa, On deformations of quintic surfaces, Invent. Math. 31 (1975), 43-85.

[K] A. G. Kouchnirenko, Polyèdres de Newton et nombres de Milnor, Invent. Math. 32 (1976), $1-32$.

[M] J. Milnor, Singular Points of Complex Hypersurfaces, Ann. Math. Studies 61, Princeton Univ. Press, 1968.

[O] М. ОкА, On the weak simultaneous resolution of a negligible truncation of the Newton boundary, Singularities (Iowa City, IA, 1986), 199-210, Contemp. Math. 90, Amer. Math. Soc., Providence, RI, 1989. Hermann, Paris, 1997.

[O2] M. Oка, Non-Degenerate Complete Intersection Singularity, Hermann, Paris, 1997.

[O3] М. ОкA, Geometry of reduced sextics of torus type, Tokyo J. Math. 26 (2003).

[O4] М. Ока, Alexander polynomial of sextics, J. Knot Theory Ramifications 12 (2003), no. 5, 619-636.

[O5] M. ОкA, Zariski pairs in Sextics, in preparation.

[O-Ph] М. Ока, D. T. Рно, Classification of sextics of torus type, Tokyo J. Math. 25 (2002), no. 2, 399-433.

[Pe] U. Persson, Configurations of Kodaira fibers on rational elliptic surfaces, Math. Zeits. 205 (1990), 1-47.

$[\mathrm{Ph}]$ D. T. Pно, Classification of singularities on torus curves of type (2,3), Kodai Math. J. 24 (2001), no. 2, 259-284.

[S-I] T. Shioda AND H. INose, On singular $K 3$ surfaces, Complex analysis and algebraic geometry, Iwanami Shoten, Tokyo, 1977, pp. 119-136.

[U] T. Urabe, Combinations of rational singularities on plane sextic curves with the sum of Milnor numbers less than sixteen, Banach Center Publ. 20 (1988), 429-456.

[W] C. T. C. WaLl, Sextic curves and quaric surfaces with higher singularities, preprint.

[Ya] Jin-Gen. YAng, Sextic curves with simple singularities, Tôhoku Math. J. 48 (1996), no. 2, 203-227.

[Yo] H. Yoshinara, Plane curves whose singular points are cusps, Proc. Amer. Math. Soc. 103 (1988), 737-740.

Department of Mathematics, Tokyo Institute of Technology

2-12-1 OH-OKayama, Meguro-Ku, ToKyo, 152-8551, JAPAN

E-mail address: ishikawa@math.titech.ac.jp 
Department of Mathematics, Hue University

32 Leloi, Hue, Vietnam

E-mail address: chanhtu@vkampen2.math.metro-u.ac.jp

Department of Mathematics, Tokyo Metropolitan University

1-1 Mimami-Ohsawa, HachioJi-Shi, ToKyo, 192-0397, Japan

E-mail address: oka@comp.metro-u.ac.jp 